\title{
Anadyoméni de Grigorios Xénopoulos
}

Des Lotophages au Banquet de Platon en passant par la Cène

Xenopoulos' Anadyomeni: from the Lotophages to Plato's Symposium

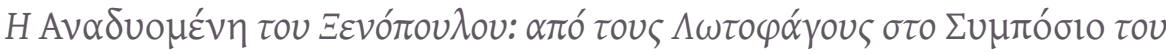

$\Pi \lambda \alpha ́ \tau \omega \nu \alpha$

\section{Gunnar De Boel}

\section{(2) OpenEdition}

\section{Journals}

Édition électronique

URL : https://journals.openedition.org/ceb/6779

DOI : $10.4000 /$ ceb. 6779

ISSN : 2261-4184

Éditeur

INALCO

\section{Édition imprimée}

ISBN : 978-2-85831-230-6

ISSN : 0290-7402

\section{Référence électronique}

Gunnar De Boel, « Anadyoméni de Grigorios Xénopoulos », Cahiers balkaniques [En ligne], Hors-série I 2016, mis en ligne le 15 mars 2017, consulté le 06 juillet 2021. URL : http://journals.openedition.org/ ceb/6779 ; DOI : https://doi.org/10.4000/ceb.6779

Ce document a été généré automatiquement le 6 juillet 2021.

\section{(c) (7) 8}

Cahiers balkaniques est mis à disposition selon les termes de la Licence Creative Commons Attribution - Pas d'Utilisation Commerciale 4.0 International. 


\section{Anadyoméni de Grigorios Xénopoulos}

Des Lotophages au Banquet de Platon en passant par la Cène

Xenopoulos'Anadyomeni: from the Lotophages to Plato's Symposium

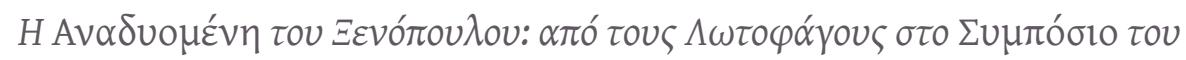

$\Pi \lambda \alpha ́ \tau \omega \nu \alpha$

\section{Gunnar De Boel}

1 L'étude critique de l'œuvre de Xénopoulos, auteur de dizaines de romans et de nouvelles publiés, comme Anadyoméni, sous forme de feuilletons dans des journaux, n'est pas à la mesure de l'immense succès populaire dont jouit toujours cet auteur, même si on a pu louer son « art consommé de la manipulation des attentes du lecteur, dans des intrigues conçues avec une précision toute mathématique " (BEATON, 1994, 100).

2 Le roman Anadyoméni en est un exemple remarquable. Les résonances littéraires de quelques repas célèbres dans des contextes mythologique, philosophique et religieux y jouent un rôle central.

3 Ce roman, paru d'abord comme feuilleton dans le journal «Ethnos» du 21 janvier au 26 mars 1923, se déroule dans le milieu aristocratique de l'île de Zante (Zakynthos), aux alentours du rattachement des îles Ioniennes à la Grèce, en 1864. Son point de départ est une prétendue légende locale qui raconte comment l'amour de deux jeunes frères, fraîchement revenus de leurs études en Italie, pour leur petite voisine Klélia, a eu pour résultat qu'on y nomme les lis sauvages des « klélias».

4 Chemin faisant, le lecteur découvre néanmoins qu'il se trouve dans une mise en pratique du Banquet de Platon, et que les épanchements romantiques de cette histoire, qui ont tellement contribué au succès tant du roman que du feuilleton qui en a été tiré pour la télévision en 1978, cachent à peine un robuste soubassement philosophique. 


\section{Anadyomeni, lis, lotus et Lotophages}

5 Le roman a pour cadre la villa "Anadyoméni », qui appartient à la famille de Klélia. Elle est appelée ainsi parce qu'elle semble surgir des vagues, tout comme Aphrodite à sa naissance. L'histoire commence avec le passage de Monsieur Mémaris avec ses deux jeunes fils, Pavlos, l'aîné, poète introverti, et Dénis, le cadet, impulsif et matérialiste, devant la villa. Dès le début, le ton de l'histoire est donné par une métaphore qui range l'amour du côté des nourritures terrestres. En effet, à la première page, le narrateur raconte comment Klélia regarde les deux jeunes gens à la dérobée par une fente dans la porte du jardin. Cette vision est comparée à la consommation d'une nourriture : «Et à travers la fente, Klélia les mangea des yeux » $(1962,327)^{1}$. Quand elle sort pour aller les saluer, la situation s'inverse, mais avec une distribution claire des rôles: "Dénis la mangeait des yeux. Pavlos la regardait à la dérobée » $(1962,330)^{2}$. Plus tard, ce seront les deux frères qui regarderont Klélia à la dérobée, par une fente de la cabine des bains de mer, au moment où elle sortira nue de la mer, en véritable « anadyomène » (1962, 353).

6 En fait, c'est l'histoire entière qui est construite de cette façon, en anneau parfait. Elle démarre avec les bourgeons de roses que Klélia offre à Pavlos, lors de cette première rencontre, pour se terminer avec les bourgeons de lis dont Pavlos fait don, avec sa vie, à Klélia. Le lis, on le verra, est le symbole crucial de ce roman : le lis sauvage, qui partage avec le lis d'eau, ou "nymphéa », la propriété de s'ouvrir le soir et de se fermer le matin. Ce lis d'eau est appelé « lotus » dans l'Égypte ancienne, où il était révéré comme symbole de la vie future. Nous y reviendrons.

7 Pour l'instant, il suffit de noter que Xénopoulos joue sur la polysémie notoire du nom de «lotus », qui recouvre plusieurs familles de plantes. L'une d'entre elles, le diospyros lotus $^{3}$ originaire du Japon et de la Corée, est effectivement cultivée dans les îles Ioniennes pour son fruit succulent, qui n'est mangé que quand il est bien mûr. Or, Klélia a justement une passion pour ce fruit, dont le côté charnel est dûment souligné : «La fleur de lotus, gamopétale, épaisse et de couleur blanche à rose, comme faite de chair " $(1962,337)^{4}$.

8 Pour être sûr que le lecteur a bien compris l'image, Xénopoulos utilise juste après le même adjectif « rose » pour les lèvres de Klélia :

Et de toute cette histoire, qui avait coulé, avec tant de grâce, tant d'innocence, des

lèvres roses de Klélia, Pavlos devinait le sens plus profond, qu'il n'arrivait pas à

concevoir clairement, comme un symbolisme indéfini.

$(1962,338)^{5}$

9 Ce symbolisme que Pavlos, «le poète qui voit des symboles partout » $(1962,431)^{6}$, n'arrive pas à saisir, ouvre un autre anneau qui ne sera fermé qu'à la fin de l'histoire. Mais cette phrase incite bien évidemment le lecteur à découvrir aussi de son côté les symboles que l'auteur a disséminés à travers le texte. En fait, par son symbolisme, ce texte s'apparente plutôt aux nouvelles qu'aux romans proprement dits de notre auteur

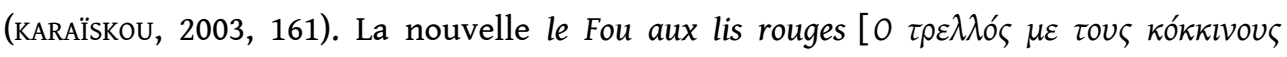
кpívouৎ], qui date de 1901, est d'ailleurs clairement le noyau d'une version alternative de notre texte, avec lequel il partage quelques éléments essentiels.

Le lotus tient donc une place dominante dans la vie de Klélia ; elle dit aux frères : « Des fruits que vous voyez là, j'ai vécu, moi ! (...) Ah, maintenant je ne mange que les lotus! » $(1962,337)^{7}$. Elle est donc, au sens le plus litéral du terme, une «Lotophage». Ainsi, 
Xénopoulos établit un lien entre l'aristocratie de Zante et le peuple mythique des Lotophages de l'Odyssée, qui ne vivent que de la cueillette de ces fruits et sombrent dans un bonheur en dehors du temps. Homère les mentionne dans le contexte d'une tempête qui fait dévier le navire d'Ulysse pendant le retour vers Ithaque. Dans la terre où ils échouent, Ulysse envoie des éclaireurs accompagnés d'un héraut, pour faire connaître son identité au roi du pays :

Alors, neuf jours durant, les vents de mort m'emportent sur la mer aux poissons. Le dixième nous met aux bords des Lotophages, chez ce peuple qui n'a, pour tout mets, qu'une fleur. (...) Mais, à peine en chemin, mes envoyés se lient avec les Lotophages qui, loin de méditer le meurtre de nos gens, leur servent des lotos. Or, sitôt que l'un d'eux goûte à ces fruits de miel, il ne veut plus rentrer ni donner de nouvelles. Tous voudraient se fixer chez ces mangeurs de lotos et, gorgés de ces fruits, remettre à tout jamais le retour.

(HOMÈRE, Odyssée, IX, 82-95, trad. V. BÉRARD adaptée) ${ }^{8}$

11 La description du pays des Lotophages par Homère ressemble à l'histoire biblique du jardin d'Éden, même s'il y a une inversion évidente: dans l'Éden, dès qu'il mange le fruit défendu, Adam est chassé du paradis et condamné à une vie de labeur, alors que chez les Lotophages au contraire, c'est le fruit qui donne accès à cet état paradisiaque, à cette vie libérée de tout labeur'. Le lien avec la vie d'oisiveté que mènent les personnages de notre histoire est d'ailleurs vite établi par le narrateur, quand il dit à propos des deux frères :

Mais l'un et l'autre faisaient le même travail maintenant: rien du tout! Ils se la coulaient douce comme la plupart des enfants de la noblesse à cette époque ; aucune occupation, aucun emploi ne leur paraissait suffisamment " aristocratique ", mis à part l'Art et la Littérature. Un moment donné, Dénis peignait. Mais bien vite il laissa tomber la peinture comme tout le reste. Pavlos, comme nous savons, écrivait des vers. Lui semblait plus stable dans son amour des arts. Peut-être deviendrait-il poète...

$(1962,392)^{10}$

12 La classe dont le portrait est fait ici est celle du «comte " Dionysios Solomos, l'aristocrate originaire de Zante et poète national de la Grèce. Le rapprochement n'est pas fortuit. On sait que, dès 1894, Xénopoulos consacrait à son grand compatriote des articles et des études, qui devaient finalement aboutir, en 1935, à la biographie romancée Riggina Léza (AмILITou, 2003, 235-37). Or, il semblerait qu'en écrivant Anadyoméni, en 1923, Xénopoulos avait déjà ce roman en tête. On constate en tout cas que plusieurs éléments du roman antérieur annoncent Riggina Léza ${ }^{11}$. Ainsi, la rivalité des frères dans Anadyoméni préfigure celle entre Dionysios et son frère Dimitris, qui, après la mort du père, a dû se charger de la gestion des domaines familiaux. Il reproche justement à son frère poète de ne rien faire :

— «D'accord, je me lève tard, mais je travaille, moi. C'est sur mes épaules que pèsent maintenant les domaines, c'est moi qui m'occupe de tout. Et je lis, je lis des livres sérieux. Demain je serai avocat, et après-demain... ah, qui sait! Mais toi ! dis, qu'est-ce que tu feras dans la vie?

- Rien! murmura Solomos entre ses dents, sans lever les yeux de son livre ».

$(1972,62)^{12}$

13 Dans Anadyoméni, le narrateur spécifie que tant que le père est en bonne santé, les fils n'ont pas besoin de s'occuper de la gestion du domaine (1962, 392-393). Mais l'identification de Pavlos à Solomos ne se limite pas à la seule appartenance à la classe aristocratique des îles Ioniennes. Rappelons que dans Anadyoméni, Pavlos, le frère aîné, est appelé régulièrement «le poète", tout comme Dionysios Solomos, qui est aussi 
l'aîné, dans le roman postérieur. En fait, il y a une parenté évidente dans la façon dont les deux poètes sont décrits. Le côté matériel de la réalité leur répugne à tous les deux ; tous les deux préfèrent aussi voir la femme idéale comme immatérielle. ${ }^{13}$ Solomos pousse cette attitude à l'extrême, en refusant de voir une femme manger, ce qui conduit son amie, Riggina Léza, à cette réflexion inquiète :

Riggina s'était déjà fait la réflexion - quand il lui avait dit qu'il ne pouvait pas l'imaginer en train de manger, qu'il la considérait comme immatérielle, comme une âme vêtue d'air. « Mais comment peut-il alors m'épouser? » s'était-elle dit.

$(1972,272-273)^{14}$

14 Or cette image sort tout droit d'Anadyoméni :

Et Pavlos se souvenait d'un article de Flammarion, qu'il venait de lire dans le « Figaro » : « Une âme vêtue d'air »... Il s'agissait d'une jeune fille de dix-sept ans, de toute beauté, que Flammarion et un ami avaient vue nue, pendant qu'elle se baignait dans la rivière. Le sage explique à son ami que ce qu'ils avaient vu, ce n'était qu'une Âme qui avait elle-même créé son corps avec des éléments pris à la nature, tous dans un état éthéré, de telle sorte que ce corps était vraiment aérien, et cette âme vêtue d'air.

$(1962,355)^{15}$

Dans Riggina Léza, ce sentiment est réciproque :

Mais Riggina l'en excusait, parce qu'au début - elle s'en souvenait - Solomos lui avait fait à peu près la même impression. Il semblait toujours immatériel - une âme, un esprit à la forme aérienne d'un ange.

$(1972,223)^{16}$

Le choix des mots rappelle à nouveau de très près le poème qu'inspire à Pavlos la vision de Klélia quand elle sort toute nue de la mer :

Pavlos voyait avec ses yeux Klélia habillée, mais avec son imagination il la voyait nue, et il esquissait, ou plutôt rêvait, un poème, un merveilleux poème qui peindrait les deux à la fois.

D'abord l'habillée, dans la verdure de la campagne (...). Et ensuite la déshabillée, l'Anadyomène, Aphrodite, la déesse. (...) Elle était dans une lumière blanche et opaque, comme celle de la perle. Et son corps mouillé ressemblait à quelque chose d'immatériel, d'aérien, de transparent, de vraiment divin. On ne voyait qu'une Âme, un Esprit, une Idée. L'Âme de la Korè, l'Esprit du Bien, l'Idée de la Beauté.

$(1962,355)^{17}$

17 La parenté entre les deux textes nous autorise à conclure à coup sûr que le personnage de Pavlos a bien été modelé sur l'image que Xénopoulos se faisait de Solomos, et que, à travers ce personnage, Xénopoulos vise la classe de Solomos, ou, du moins, ce qu'elle représente quelque quarante ans après ce séjour de Solomos à Zante. Le moins qu'on puisse dire, c'est que Xénopoulos, en établissant un lien entre cette classe et les Lotophages, semble suggérer que cette aristocratie a perdu le contact avec la réalité.

\section{Du Banquet}

Mais l'intertextualité ne s'arrête pas là, tant s'en faut ! L'auteur ajoute habilement à la « lotophagie » homérique des indices qui renvoient avec de plus en plus d'insistance au Banquet platonique, ce texte fondateur de la réflexion occidentale sur l'amour, qui raconte les discours tenus par quelques convives, dont Socrate, au banquet qu'offre Agathon lors de la victoire de sa première tragédie, en 416 av. J.-C. Xénopoulos fait le lien entre les lotus et l'amour de la façon suivante : «Et elle se défoulait en mangeant 
des lotus. Mais bientôt même eux lui paraissaient fades. Elle ne voulait que les lotus de Dénis... » $(1962,397)^{18}$ c'est justement le poème de Pavlos mentionné déjà : on peut penser qu'au moment où Klélia devient "anadyomène ", elle naît en tant qu'Aphrodite, et, comme dans le Banquet, l'Amour (Éros) est engendré le jour de la naissance d'Aphrodite (203c). En revanche, il est indéniable que ce poème renvoie au passage du Banquet ${ }^{20}$ de Platon où il est question de l'idée de la beautée ${ }^{1}$ :

Quelle idée nous faire dès lors, ajouta-t-elle, des sentiments d'un homme à qui il serait donné de voir le beau en lui-même, dans la vérité de sa nature, dans sa pureté, sans mélange ; et qui, au lieu d'un beau infecté par des chairs humaines, par des couleurs, par mille autres sornettes mortelles, serait au contraire en état d'apercevoir, en lui-même, le beau divin, dans l'unicité de sa forme?

(211e, trad. L. ROBIN $)^{22}$

21 rapporte ici la théorie de Diotime, la sage étrangère d'Arcadie, qui enseigne qu'on accède à cette «idée de la beauté » par paliers, à partir justement des beaux corps des jeunes gens :

Car c'est là justement le droit chemin pour accéder aux choses de l'amour (...), de partir des beautés de ce monde et, avec cette beauté-là comme but, de s'élever continuellement, en usant, dirais-je, d'échelons, passant d'un seul beau corps à deux, et de deux à tous, puis des beaux corps aux belles occupations, ensuite des occupations aux belles sciences, jusqu'à ce que, partant des sciences, on arrive pour finir à cette science que j'ai dite, science qui n'a pas d'autre objet que, en ellemême, la beauté dont je parle, et jusqu'à ce qu'on connaisse à la fin ce qui est beau par soi seul.

(211b-c, trad. L. ROBIN $)^{23}$

Il faut peut-être préciser que, si chez Platon le premier palier est constitué par la pédérastie (211b), cela n'est évidemment pas le cas chez Xénopoulos.

Une deuxième référence irréfutable au Banquet de Platon suit quand Klélia boude pendant quelque temps le jeune matérialiste Dénis et se met à discuter de philosophie avec son frère, le poète : « Un jour, elle questionna Pavlos à propos de l'Amour » (1962, $372)^{24}$. Cela rappelle la façon dont Socrate introduit le discours de Diotime : «On doit (...) expliquer d'abord ce qu'est l'Amour lui-même, sa nature et ses attributs, et ensuite ses œuvres » (201d-e, trad. L. ROBIN $)^{25}$. Klélia développe sa question en faisant référence aux grandes histoires d'amour dont abondent l'histoire et la mythologie, comme le fait Phèdre dans le texte de Platon. Xénopoulos se borne à remplacer les exemples platoniques d'amoureux qui ont sacrifié leur vie pour l'objet de leur amour : Alceste, Orphée, Achille, par l'exemple d'un grand amour médiéval, celui d'Abélard et d'Héloïse $(1962,372)$. Ensuite, il n'hésite pas à mettre dans la bouche de Klélia le mythe des êtres sphériques, coupés en deux, tel que le raconte Aristophane dans le Banquet (189e-193c) :

J'ai lu quelque part une tradition comme quoi Dieu aurait créé les âmes deux par deux, jumelles, et il les aurait séparées et dispersées de par le monde. Chaque âme essaie donc de trouver sa sœur, sa moitié. Et le grand, l'éternel amour naît quand deux âmes jumelles réussissent à se rencontrer.

$(1962,372)^{26}$ 
Après ces annonces, le roman devient effectivement l'histoire non pas d'un seul, mais de trois "banquets", dont le premier et le troisième se passent dans les ruines d'un monastère où il n'y a plus qu'un seul moine-prêtre, le père Ignatios, un ami, que la première fois les deux familles vont visiter sans l'avoir prévenu.

Les repas au monastère empruntent plusieurs éléments à la mise en scène du Banquet de Platon, comme justement le fait de s'y rendre sans y avoir été invité. Ainsi, Aristodème se laisse convaincre par Socrate, qui est convié au repas chez Agathon, de l'y accompagner (174b), ce qui n'empêche pas Agathon de l'accueillir chaleureusement (174e). De la même façon, lors du premier repas, la compagnie arrive à l'improviste chez le père Ignatios, qui insiste pour leur offrir un excellent repas quand-même :

Des vins qui ont de la bouteille, de la poutargue fraîche, du caviar cendré - le meilleur ! - et de la mostarda douce pour le rôti, faite de coings aux grains de moutarde, quelque chose de merveilleux !

$(1962,379)^{27}$

Il leur vante toutes ces nourritures comme « exquises $»^{28}$, et raconte la "performance d'un sage moine fin gourmet ", ${ }^{29}$ qui sur le mètre de l'Hymne des Salutations avait fait un louange à toutes les bonnes nourritures que produisent la terre et la mer grecques. D'après le modèle de la première stance, citée par le narrateur : «J'ouvrirai la bouche/ et elle sera remplie de l'esprit./(...)/je chanterai dans la joie/toutes ces merveilles $»^{30}$, cela donne: «Des olives d'Amphissa,/et de la poutargue jaune/de Mésolongi/du vin verdéa du pays,/(...) des choses exquises !» $(1962,379)^{31}$.

Or ce moine fine bouche ne semble être personne d'autre que Kaisarios Dapontes (1714-1784), du monastère Xiropotamou au mont Athos, qui a mis tout ce qu'il a vu, entendu ou lu en vers (PoliTIS, 1991, 92), puisque celui-ci, dans un texte paru en 1778, parodie le même hymne d'exactement la même façon, en terminant également tous les tropaires de la première stance avec « des choses exquises » : « du vin de Skopélos,/du koumandaréa de Chypre,/du muscat de Samos,/et plusieurs vins de France/(...) des choses exquises $(1991)^{32}$. Xénopoulos, une fois de plus, et sans crier gare, se fait « intertextuel » de la façon la plus érudite ${ }^{33}$.

2 Tout le monde se pique au jeu, et essaie de faire rentrer les nourritures les plus exquises les unes que les autres dans le mètre. Pavlos le poète tient bien sûr parfaitement le mètre, mais l'immatérialiste qu'il est est bien vite à court d'inspiration quant aux nourritures terrestres. Dénis, par contre, déborde d'imagination sur ce sujetlà, mais il commet tout le temps des fautes contre la métrique $(1962,380)$. Le repas se déroule donc en suivant le modèle des symposia antiques, avec des jeux et des chants (BASLEZ, 1998, 278).

Mais l'intérêt de ce passage ne se limite peut-être pas au caractère facétieux de la parodie, étant donné que dans les tropaires suivants du texte original, qui ne sont plus cités dans le roman, non seulement la Sainte Vierge est comparée à un lis parfumé, mais en plus un rôle décisif lui est attribué dans la résurrection des morts : «Ô trésor de pureté, salut! Par ton intercession nous sommes ressuscités des morts; salut, lis parfumé, Notre Dame, qui embaumes les fidèles ; encens odorant, huile précieuse ${ }^{34}$. La métaphorique est complétée par la comparaison de la Vierge à une table vivante qui porte le pain de la vie: «Salut, Toi, champ non labouré, qui as fait pousser l'épi divin, Toi, table animée, qui porte le pain de la vie. Salut, Notre Dame, source intarissable d'eau vivante $»^{35}$. 
allusion cachée à l'eucharistie est ensuite explicitée dans la scène qui réunit les deux familles pour un deuxième " banquet », qui est en fait le dernier repas de la saison dans la villa "Anadyoméni », avant qu'elles ne rentrent en ville pour y passer l'hiver :

Tout le repas était local de $a$ à $z$. Le pressoir d'Ypsolithos fournissait l'huile nouvelle pour la fameuse chicorée sauvage, et Giouanes avait rapporté de la ville du saumon $^{36}$, ce poisson rouge salé qui l'accompagne traditionnellement. Après il y avait des poulets, cuisinés eux aussi à la paysanne ; c'était un plat " pour des rois", d'après le comte Giorgis. Et pour finir du fromage frais et des fruits choisis, surtout des melons mûris dans la terre sablonneuse - le seul fruit qui se compare aux lotus, d'après Klélia - et du raisin noir, avec un arôme de fraises, la folie! Et du vin, alors là ! Un verdéa embouteillé, de la cave de l'Anadyoméni, avec des toiles d'araignée aux bouchons et des inscriptions jaunies : 1848, 1849, 1850... de la main de feu le vieux Landos le sage.

$(1962,418)^{37}$

Ces aristocrates ne mangent que les bonnes choses de leur terroir: une référence évidente, d'abord, à l'idéologie terrienne qui caractérise cette classe. Mais là encore, le lotus s'immisce: la comparaison des melons exquis avec le lotus souligne la caractérisation, dès le début du texte, de cette classe comme « lotophage », avec tout ce que cela implique.

Ensuite, ce repas est explicitement comparé à la dernière cène, qui a donné le modèle de l'eucharistie. À l'origine, d'ailleurs, l'eucharistie n'était que la forme chrétienne du symposion antique, avec la même division entre repas et symposion proprement dit, pendant lequel on buvait du vin, on chantait et on discourait (ALIKIN, 2009, 57). Même la symbolique nuptiale de l'eucharistie (MCGUCKIN, 2011, 292), est exploitée :

Dis donc, c'est la dernière cène ! dit $\mathrm{M}$. Memaris à un moment donné.

Et vraiment, ce repas avait un certain mystère, une certaine poésie. Au nom d'une vieille coutume, d'une tradition vénérable, il rassemblait autour de cette table les deux familles amies que réuniraient bientôt les liens indissolubles.

$(1962,418)^{38}$

Mais évidemment, si ce dernier repas à la campagne, comme la dernière cène, renvoie à une fin, c'est qu'il prélude en réalité à une mort :

comme leur humeur, qui était gaie, mais aussi mélangée à la mélancolie de l'automne et au chagrin d'une fin.

Cela, c'était Pavlos qui le ressentait le plus, même si à table il était le plus joyeux de tous. À le voir, à l'entendre, comment soupçonner que ce jeune homme avait la mort dans l'âme?

$(1962,418)^{39}$

La comparaison de ce repas avec la dernière cène dresse donc le décor pour la suite de l'histoire, qui pour la première fois ici est annoncée aussi clairement.

Enfin, il y a un second - et dernier - repas au monastère. Le père Ignatios, prévenu à l'avance cette fois-ci, a eu le temps de préparer d'excellentes nourritures : « des tripes d'agneau arrosées d'une sauce à l'œuf et au citron, de l'agneau à la broche, des boyaux tressés, et ainsi de suite » $(1962,436)^{40}$.

Ce repas présente lui aussi plusieurs points de ressemblance avec le Banquet de Platon. Lorsque celui-ci commence, la plupart des participants sont encore sous le coup de la beuverie de la veille (176a-b), et quand Alcibiade arrive, il ne cesse de prétendre qu'il est déjà ivre mort. Mais Socrate ne se laisse pas abuser : "Je soupçonne, Alcibiade, ditil, que tu as été sobre aujourd'hui » (222c) ${ }^{41}$. 

l'excellent repas que le moine leur a servi ; mais Pavlos encore plus, ou du moins, c'est l'impression qu'il veut donner aux autres : «Et ils le croyaient ivre, alors qu'il ne l'était pas du tout $»(1962,437)^{42}$. dessein, qui est, d'après le commentaire de Sykoutris, le suivant :

Alcibiade feint d'être ivre, alors qu'il est absolument sobre; son but est bien calculé : il veut semer la discorde et annuler les liens, qui commencent à se former entre Agathon et Socrate. (...) et l'épisode se termine sur une belle image : Socrate, l'éternel amant - l'éternel objet d'amour, entouré par les deux jeunes gens les plus beaux et les plus glorieux du jour - un trio d'Amour.

$(1934,181-2)^{43}$

On pourrait dire que dans Anadyomeni, l'ivresse feinte de Pavlos a un but exactement inverse : si elle sert à cacher son exaltation devant sa résolution fatale, celle-ci résulte du fait que lui accepte le choix de Klélia pour son frère, un choix qui détruit le trio qu'ils formaient avant : «Ils étaient devenus deux plus un, ou deux contre un » (1962, 391). ${ }^{44}$

40

Cette ivresse feinte conduit en ligne droite au dénouement du roman: la deuxième cueillette de lis sauvages. La première avait été le fait de Dénis, qui avait réussi, en descendant une pente très ardue, au risque de sa vie, à gagner de façon héroïque l'amour de Klélia. Mais les lis qu'il avait cueillis alors, et que Klélia avait plantés ensuite, n'avaient pas pris, malgré le désir ardent de celle-ci de voir couverte la côte en bas de la villa de ces fleurs à la couleur de ses yeux, qui lui étaient devenues si chères au cœur (1962, 414, 443). En effet, par la comparaison explicite des lis avec les «choses exquises » dont le père Ignatios gratifie ses hôtes $(1962,390,431)$, ils sont clairement rangés du côté des nourritures terrestres, et particulièrement des lotus, dont Klélia raffole. C'est cette passion de Klélia qui donne à Pavlos l'idée d'aller lui aussi en cueillir maintenant, en haut du ravin dont son frère n'était sorti vivant que par miracle. Ainsi, il déguisera son suicide en accident, tout en mettant en pratique l'un des thèmes du Banquet de Platon : celui de l'amant qui meurt pour l'être aimé. Au fond, c'est également l'issue de la dernière cène. Mais ni dans le Banquet ni dans l'Évangile, la mort n'a le dernier mot. Les lis que Pavlos avait réussi à déraciner revivront bel et bien :

Soudain ils ont repris du poil de la bête, ils se sont mis à vivre. Et le troisième jour, le soir, les petits lis bleus se sont joliment ouverts. Ils avaient pris! (...) Mais oui, ils avaient pris, des bourgeons poussaient. Un miracle, un véritable miracle.

$(1962,442)^{45}$

41 Ainsi, l'anneau de la composition se referme : les bourgeons que Klélia avait offerts à Pavlos au début de l'histoire, lui reviennent à la fin pour combler son désir le plus cher. Et cela se passe évidemment au troisième jour, comme dans les Évangiles :

Il n'est pas ici ; mais il est ressuscité. Rappelez-vous comment il vous a parlé, quand il était encore en Galilée : il faut, disait-il, que le Fils de l'homme soit livré aux mains des pécheurs, qu'il soit crucifié, et qu'il ressuscite le troisième jour.

(Luc, 24, 6-7, trad. Bible de Jérusalem) ${ }^{46}$

Ici, ce ne sont évidemment que des fleurs, et non Pavlos lui-même qui ressuscite. En effet, le propos de Xénopoulos n'est pas chrétien. N'a-t-il pas dit expressément que ni le pope, ni ses hôtes ne sont croyants $(1962,378,380)$ ? La vie future qui est esquissée ici ne correspond pas aux conceptions chrétiennes, mais bien plutôt à celles du Banquet, et plus particulièrement à celles de l'une de ses sources principales, le poète-philosophe 
sicilien Empédocle. Victor Cousin, l'auteur de l'édition avec traduction et commentaire du Banquet de Platon qui pourrait être celle que Xénopoulos utilisait (cf. note 21), remarque même: «Lorsque Platon parle des poètes, il est d'autant plus juste de supposer qu'il pense à Empédocle» $(1831,460)$. Or, Pavlos est présenté systématiquement comme poète, encore et surtout au moment où le narrateur suggère que son projet de suicide prend forme définitivement $(1962,431)$.

Ce projet avait été évoqué pour la première fois au moment où il devenait évident que les lis arrachés par Dénis ne prenaient pas. Devant la tristesse de Klélia, tout d'un coup Pavlos a de nouveau une "idée indéfinie ", comme celle qu'il avait eue quand Klélia parlait de sa passion pour les lotus. Mais maintenant cette idée, dont il n'entrevoit qu'à peine les contours, le remplit de joie :

Mais soudain une joie sauvage éclata dans les yeux de Pavlos... À quoi pouvait-il bien penser?...

Lui-même ne savait pas bien le dire. C'était tellement vague, tellement indéfini !... Mais même ainsi cela lui procura une jubilation indicible et étrange. Peut-être parce qu'il pressentait que cette idée vague allait s'intensifier, cette idée indéfinie allait se clarifier et tout doucement le dominer complètement...

Et ce serait beau, et grand!

$(1962,415)^{47}$

L'acte qui commence à s'imposer à lui sera donc quelque chose de "grand »: comme qui dirait un «beau travail $»^{48}$, un de ceux qui valent, d'après Platon (209e), un culte à leur auteur. Dans le contexte philosophique tel qu'il a été préparé par le narrateur, il me semble évident que la mort de Pavlos, qui se jette dans un ravin, est inspirée par la mort d'Empédocle, qui se jeta dans l'Etna. C'est d'ailleurs précisément l'exemple qui vient à l'esprit d'Horace, quand il veut illustrer un acte de poète par excellence, dans son Art Poétique :

Je raconterai la légende du poète de Sicile, Empédocle, qui voulut se faire passer pour un dieu, et, de sang-froid, se précipita dans les flammes de l'Etna. Le poète a la liberté et le droit de se donner la mort ; le sauver malgré lui, c'est le tuer. Ce n'est pas la première fois qu'on en voit agir ainsi : sauvez-le, il ne redeviendra pas un simple mortel, et ne renoncera pas à la gloire d'une mort fameuse.

(vers 464-469, trad. de Fr. RICHARD) ${ }^{49}$

Les conditions de ce suicide, telles que racontées par Diogène Laerce dans sa vie d'Empédocle, rappellent d'ailleurs cette excursion bucolique de Pavlos et de sa famille :

Après le repas, quelques uns se retirèrent pour se reposer, quelques autres se mirent sous les arbres d'un champ voisin, d'autres s'en allèrent où ils voulurent; Empédocle se tint dans la place qu'il avait occupée pendant le repas; le lendemain chacun s'étant levé, il n'y eut qu'Empédocle qui ne parut point.

$(8,2,68)^{50}$

L'interprétation empédocléenne s'impose d'autant plus ici qu'Empédocle est célèbre aussi pour sa théorie de la métempsycose, selon laquelle les hommes peuvent se réincarner même en des animaux ou des plantes. C'est encore Diogène Laerce qui lui attribue cette conception :

Il estimait que l'âme revêt toutes sortes de formes de plantes et d'animaux. Il assure : j'ai été jadis un garçon et une fille, un buisson et un oiseau et un poisson écailleux de la mer.

$(8,2,77)^{51}$

En effet, ce roman s'achève sur une suggestion de réincarnation, puisque l'amour du jeune homme ressuscite sous la forme de lis sauvages: 
Les lis couvraient le domaine complètement, comme si c'était l'amour du jeune homme lui-même qui débordait de son cœur, au moment où il donnait sa vie pour elle...

$(1962,444)^{52}$

D'ailleurs, même abstraction faite de cette réincarnation, le geste de Pavlos, qui a donné lieu à une véritable légende dans l'île $(1962,444)$, lui fait obtenir la gloire et l'immortalité : la récompense qui attend, toujours d'après Platon dans le Banquet, les poètes et autres auteurs de «beaux travaux ", qui n'ont rien de la charité chrétienne, mais ressemblent plutôt au « quelque chose de grand » que mentionnait Pavlos :

Il n'est point d'homme qui ne préfère de tels enfants aux enfants des hommes s'il vient à considérer, avec une noble jalousie, la renommée et la mémoire immortelle que garantissent à Homère, à Hésiode et aux grands poètes leurs œuvres, étant elles-mêmes immortelles.

(Banquet, 209c-d, trad. cousin adaptée) $)^{53}$

Quoi qu'il en soit, Pavlos remporte ainsi une victoire postume sur son frère. Klélia se rend finalement à l'évidence que son amour à lui était bien plus profond que celui de Dénis :

Et elle disait souvent à Dénis : "J'ai planté les tiens aussi, à ce moment-là, tu te souviens? Mais ils n'ont pas pris. Ceux de Pavlos, par contre, nous ont inondés. Lui les a arrachés en y mettant tout son cœur ! Lui m'aimait plus que toi ! » $(1962,443)^{54}$

L'œuvre du poète, inspirée par son amour véritable, a donc réussi à faire sauter les limites de la vie.

\section{Conclusion}

51 Pour conclure, on peut dire que les lis sauvages, qui s'ouvrent le soir et restent ouverts toute la nuit, deviennent dans ce roman des symboles de la vie après la mort, tout comme le lotus égyptien, avec lequel ils partagent cette caractéristique. Ils y forment le principe de l'«impression unifiée » que Xénopoulos emprunte à Poe: une idée qui traverse, et unifie, le texte du début à la fin, de façon allusive, en raccordant les différentes parties (KARAÏsKOU, 2003, 163-164).

L'auteur utilise, me semble-t-il, ces lotus à deux niveaux. D'une part, ils convergent, en tant que symbole, avec l'image de la dernière cène pour signifier une résurrection, ou du moins un retour au monde de cette classe de Lotophages dont parle ce roman depuis le début. Les lis, qu'aimait tellement Klélia, sont, à la fin, aimés démocratiquement par tous les jeunes gens et les jeunes filles de l'île, pour qui toute cette époque est devenue légendaire.

D'autre part, ce roman parle bien sûr aussi de la mémoire immortelle qui vient récompenser le sacrifice du poète platonique, qui a entrevu l'idée de la beauté à travers une beauté passagère et, tout compte fait, assez mesquine. Ce n'est pas le moindre mérite de Xénopoulos d'avoir su faire pleurer ses publics en leur proposant une défense et illustration du Banquet de Platon... 


\section{BIBLIOGRAPHIE}

ALIKIN Valeriy Alexandrovich, 2010, The Earliest History of the Christian Gathering: Origin, Development and Content of the Christian Gathering in the First to Third Centuries, Leyde: Brill.

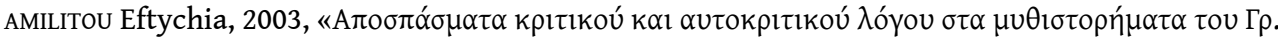

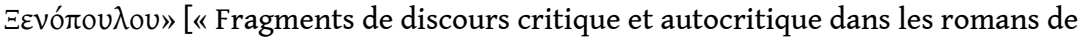

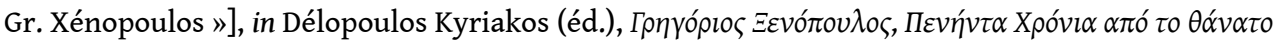
$\varepsilon v o ́ s \alpha \theta \alpha ́ v \alpha \tau o v$ [Grigorios Xénopoulos, cinquante ans après la mort d'un immortel], Athènes : ELIA, p. 217-246.

BASLEZ Marie-Françoise, 1998, Bible et histoire : judaïsme, hellénisme, christianisme, Paris : Gallimard. BEATON Roderick, 1994, An Introduction to Modern Greek Literature, Oxford: Clarendon Press. cousin Victor, 1831, Phèdre, Ménon, le Banquet : œuvres de Platon traduites par Victor Cousin, Tome sixième, Paris : Pichon et Didier.

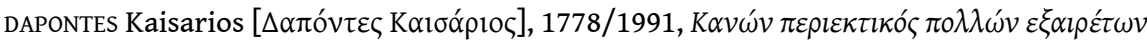


beaucoup de choses exceptionnelles constatées dans de nombreuses villes \& îles \& nations \& animaux (1778)], édité par G. P. Savvidis, Athènes : Leschi (cité d'après http://users.uoa.gr/ nektar/orthodoxy/gerontikon/kaisarios_dapontes_logoi_panhgyrikoi/ 13_kanwn_exairetwn_pragmatwn.htm ).

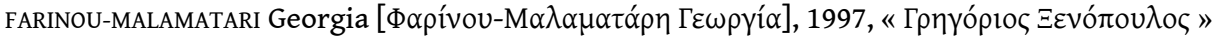

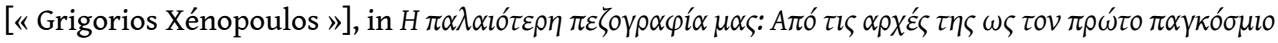
$\pi o ́ \lambda \varepsilon \mu_{0}$ [Notre prose ancienne : des débuts jusqu'à la Première Guerre mondiale], Tome 9 (19001914), Athènes : Sokolis, 1997, p. 288-377.

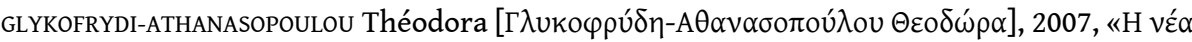

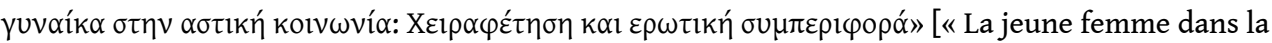

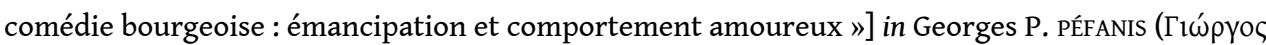

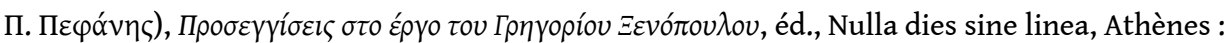
Fondation Kostas et Éléni Ourani, p. 65-101.

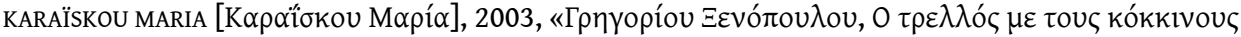

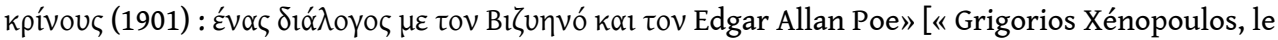
fou aux lis rouges (1901) : un dialogue avec Vizyinos et Edgar Allan Poe »], in Kyriakos DéLopoulos

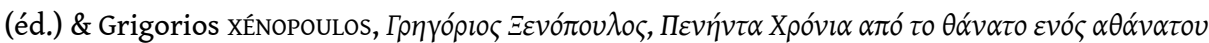
[Cinquante ans après la mort d'un immortel], Athènes : ELIA, p. 159-179.

MCGUCKIN John Anthony, 2011, The Orthodox Church: an Introduction to its History, Doctrine and Spiritual Culture, Oxford: Wiley-Blackwell.

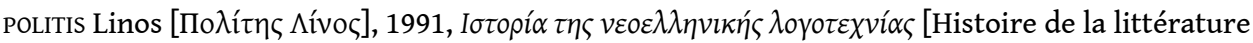
néohellénique], Athènes : MIET.

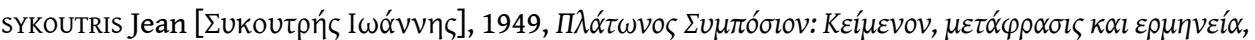

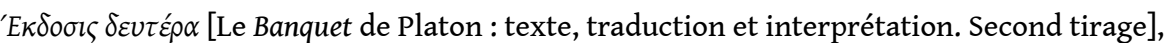
Athènes : Estia. 


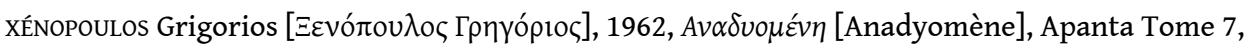
Athènes : Biris, p. 323-444.

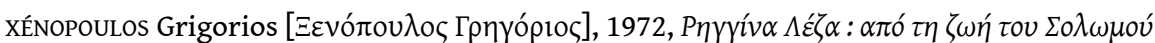

[Riggina Léza : scènes de la vie de Solomos], Apanta, Tome 10, Athènes : Biris, p. 11-304.

\section{NOTES}

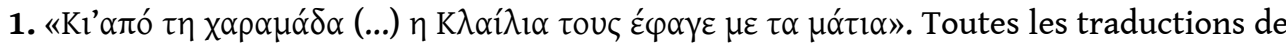
passages des romans de Xénopoulos sont de ma main. Leur seule ambition est d'aider le lecteur non-hellénophone à comprendre le texte grec.

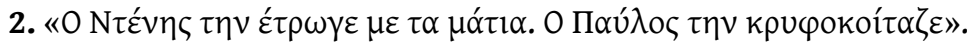

3. <https://el.wikipedia.org/wiki/ $\Lambda \omega \tau o ́ \varsigma>$

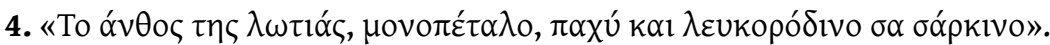

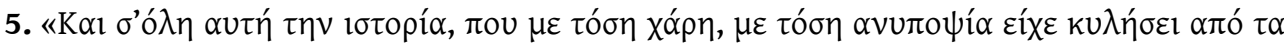

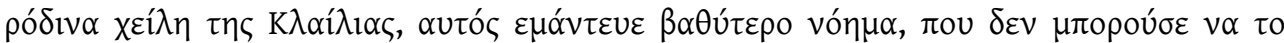

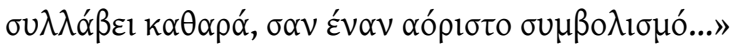

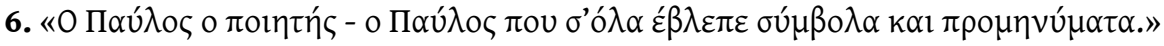

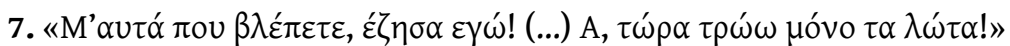

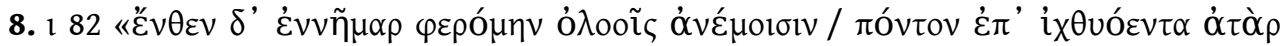

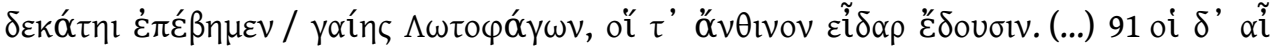

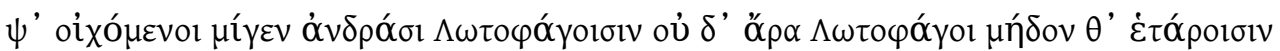

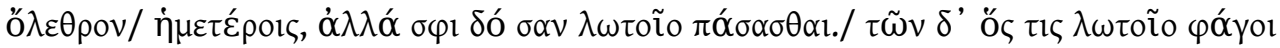

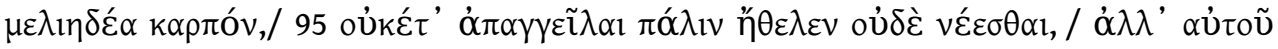

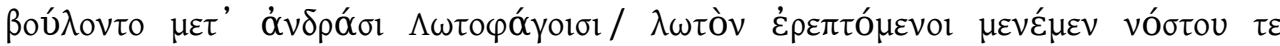

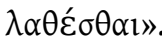

9. C'est précisément le thème du poète anglais Alfred TENNYSON dans "The Lotos-Eaters" (1832). Mais le roman de xéNOPoulos ne semble pas contenir de références à ce célèbre poème.

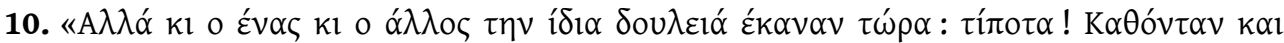

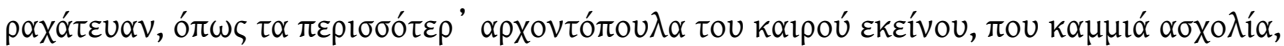

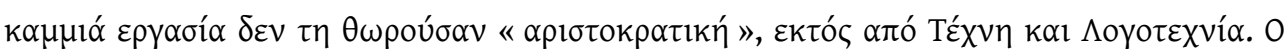



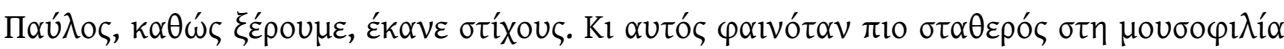

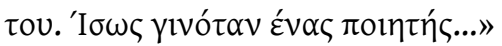

11. Déjà le nom de Dénis renvoie bien sûr à « Dionysios ", même si c'est son frère Pavlos qui correspond à Solomos, et que Dénis serait plutôt en tout le contraire du poète national. Mais cette façon de prendre le contre-pied du modèle est aussi une manière de le citer ; on retrouvera plus bas cette inversion dans la façon dont Xénopoulos utilise le motif de l'ivresse dans le Banquet de Platon.

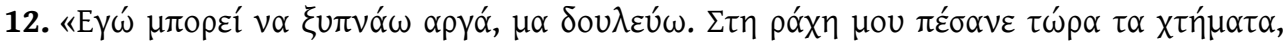

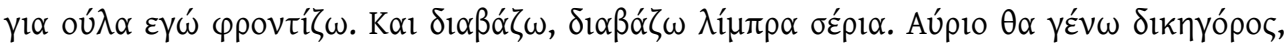

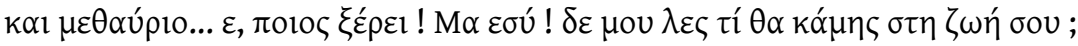




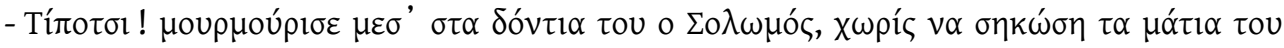

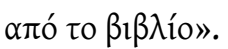

13. L'amour éthéré, immatériel est souvent opposé à l'amour charnel dans l'œuvre de Xénopoulos (FARINOU-MALAMATARI, 1997, 305 ; GLYKOFRYDI-ATHANASOPOULOU, 2007, 88).

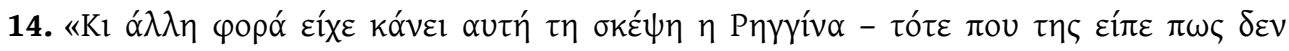

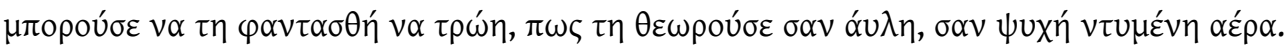

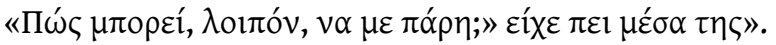

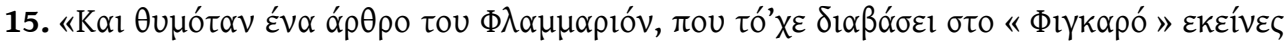

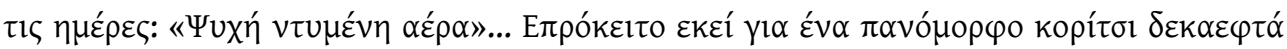

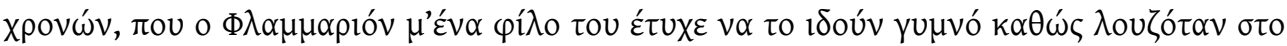

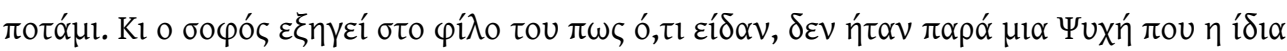

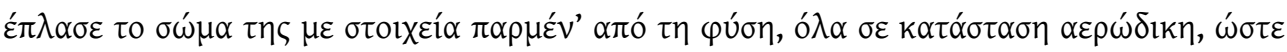

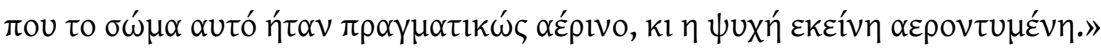

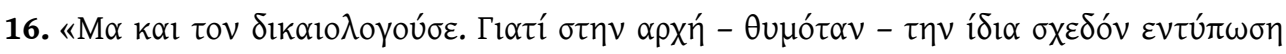

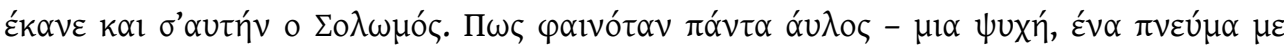

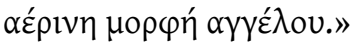

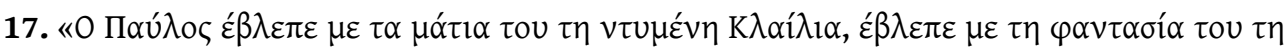

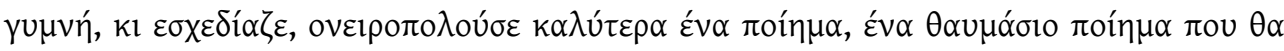

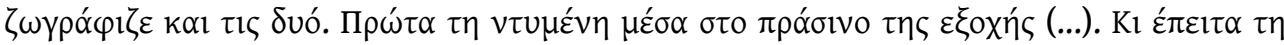

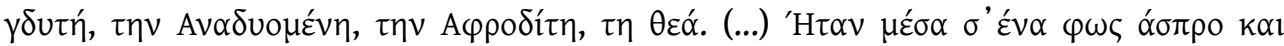

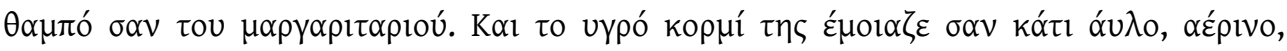

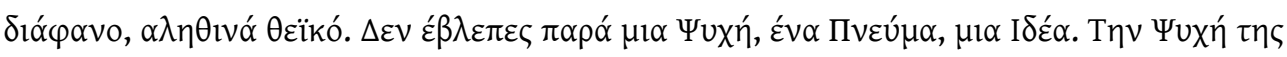

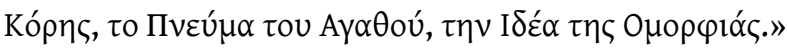

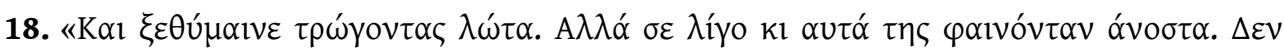

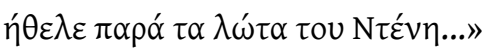

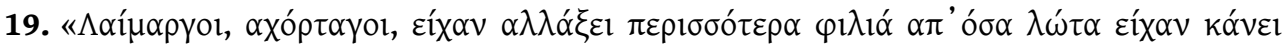

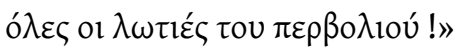

20. L'intérêt que portait Xénopoulos au Banquet est démontré aussi, en plus de tous les points de convergence que nous relèverons, par son compte rendu de la célèbre première édition grecque, avec traduction et commentaire, de Sykoutris (Néa Estia, 183, 1934, 708-709).

21. Il est vrai que Platon n'utilise pas lui-même le terme «idée de la beauté », mais qu'il parle plutôt du "beau en lui-même ». Néanmoins, les commentaires, dont celui de Victor cousin $(1831,455,466)$, parlent systématiquement de l'«Idée de la Beauté ». Or, en 1923, quand Xénopoulos publie Anadyoméni, il n'y a pas encore d'édition grecque du texte original du Symposion. De même, celle de Léon Robin pour la collection Budé ne sortira qu'en 1929. Il est à mon avis probable que Xénopoulos utilisait cette édition de Cousin, qui sera d'ailleurs encore citée dans l'édition de Sykoutris, qui date de 1934

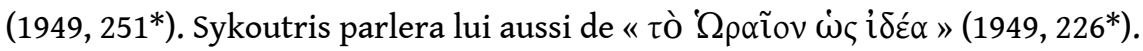

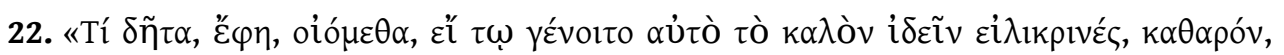

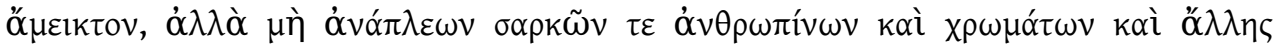

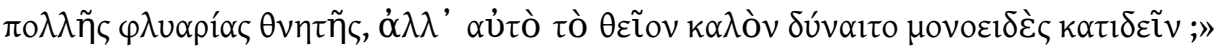

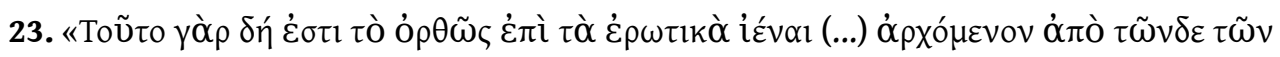

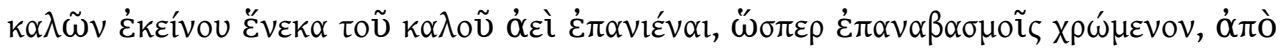

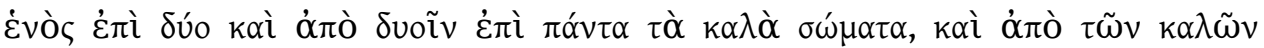




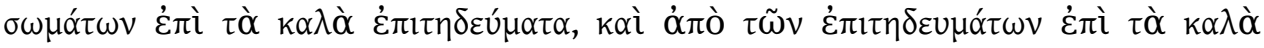

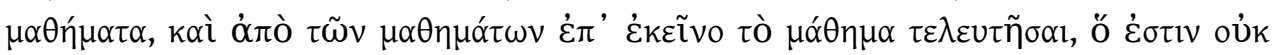

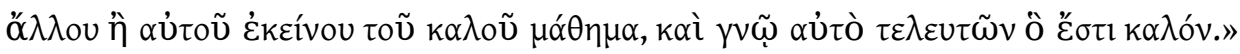

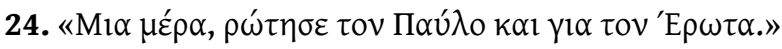

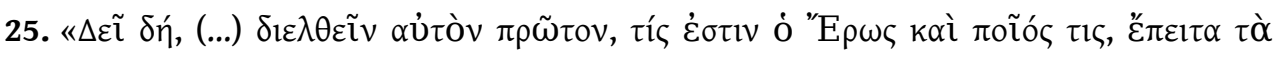

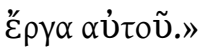

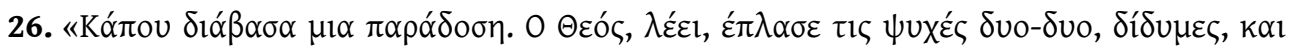

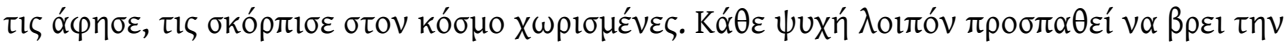



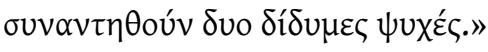

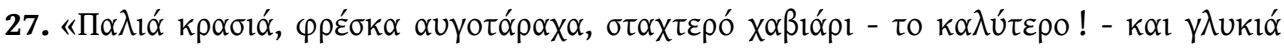

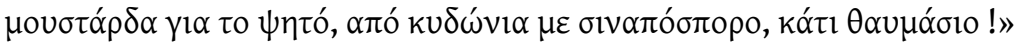

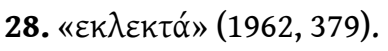



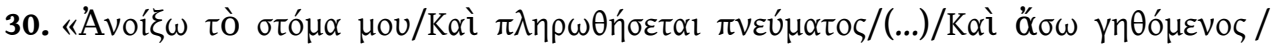

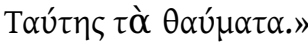

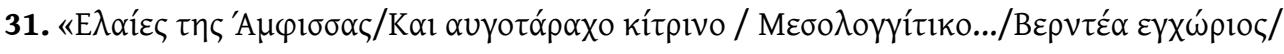

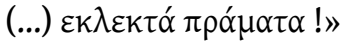

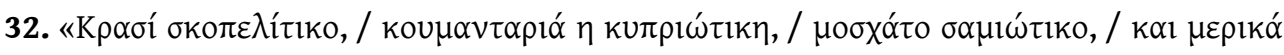

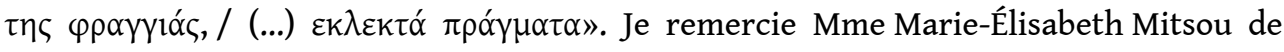
m'avoir signalé ce texte de Kaisarios Dapontes.

33. Mme Solange Festal-Livanis me signale que cette coutume existe toujours dans l'île. Ce serait intéressant de savoir s'il y a un lien avec Dapontes et Xénopoulos, et, si oui, dans quel sens il irait.

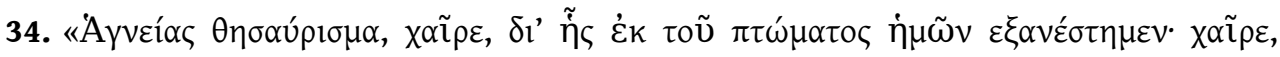

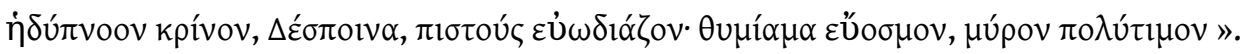

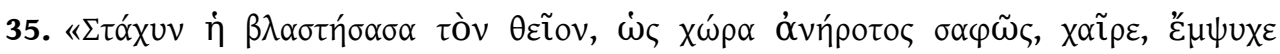

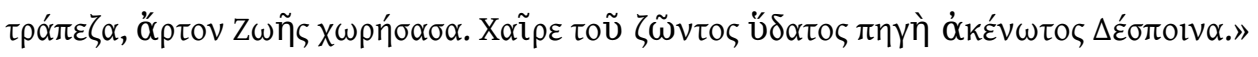

36. Dans Riggina Léza, Xénopoulos revient à plusieurs reprises sur le fait qu'à Zante le nom de famille "Solomos» (par ailleurs homonyme du mot pour «saumon»), était prononcé « Solémos », comme c'est le cas du poisson ici.

37. «'O

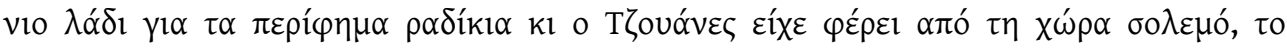

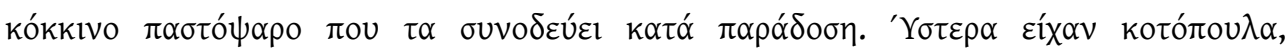

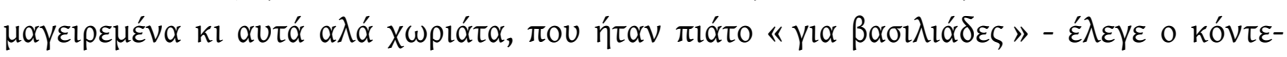

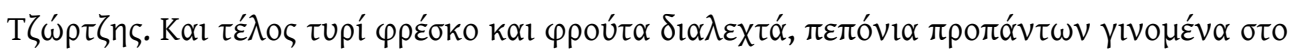

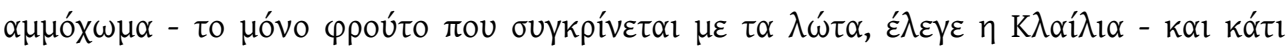

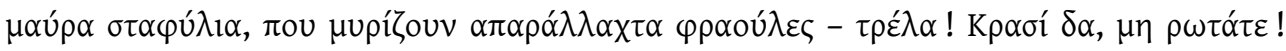

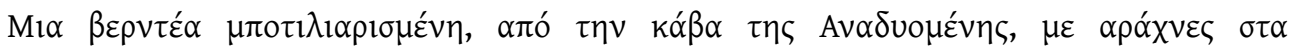

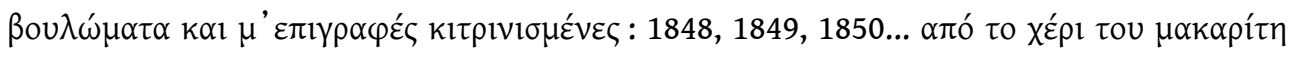

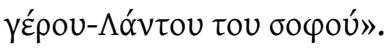

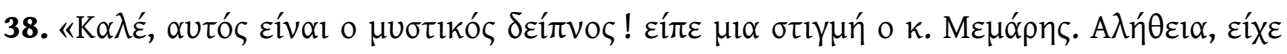

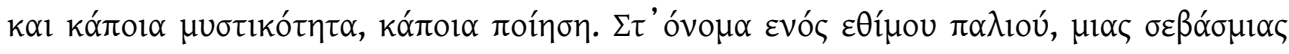




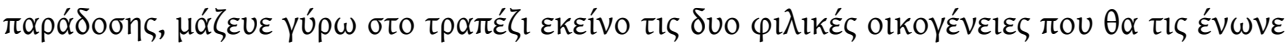

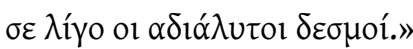

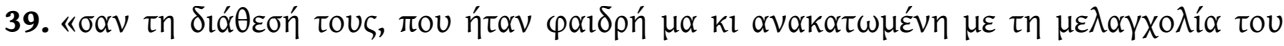

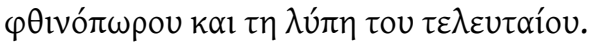

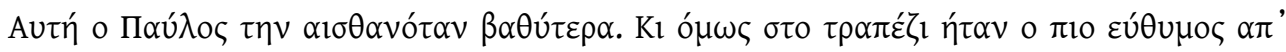

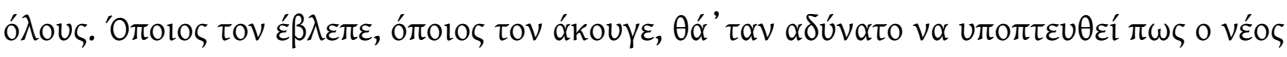

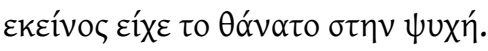

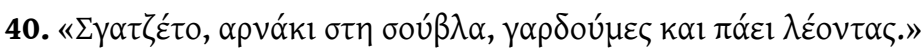

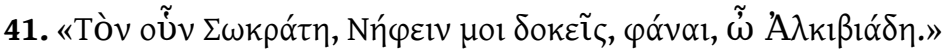

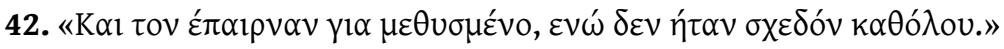

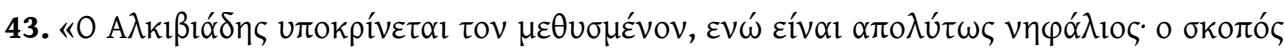

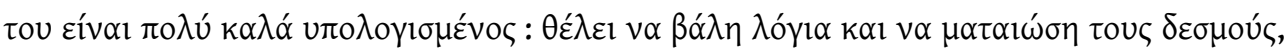

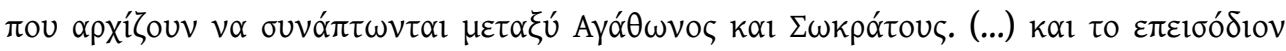

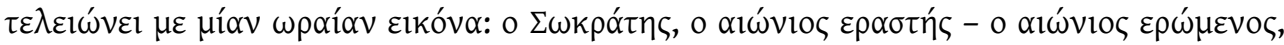

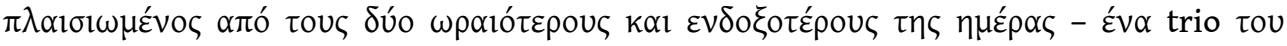
'Е $\rho \omega \tau$ то५.»

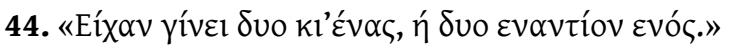

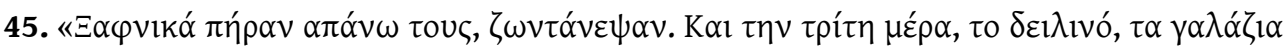

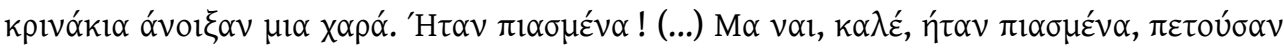

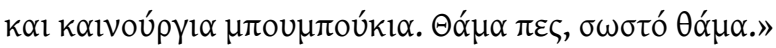

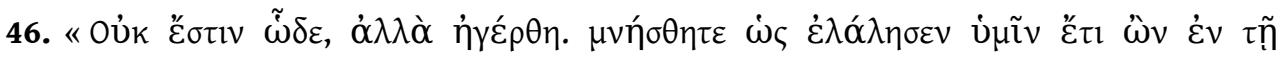

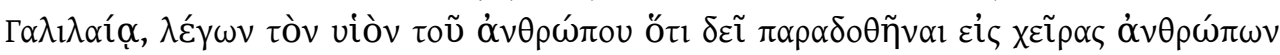

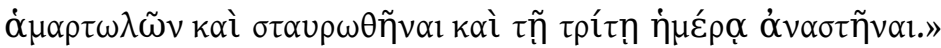



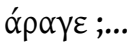

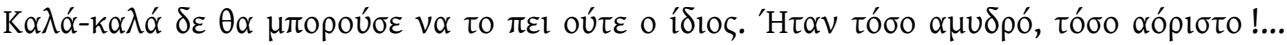



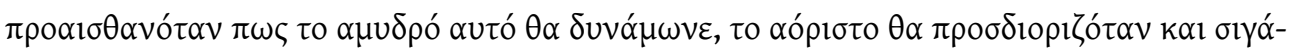



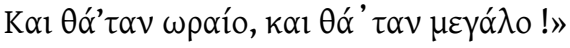

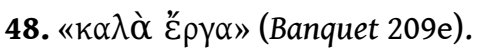

49. Siculique poetae/narrabo interitum. "deus immortalis haberi/465 dum cupit Empedocles, ardentem frigidus Aetnam/insiluit. sit ius liceatque perire poetis :/invitum qui servat, idem facit occidenti./nec semel hoc fecit nec, si retractus erit, iam/fiet homo et ponet famosae mortis amorem.

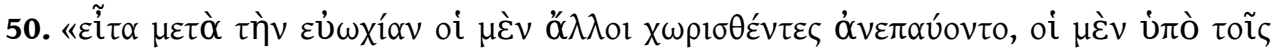

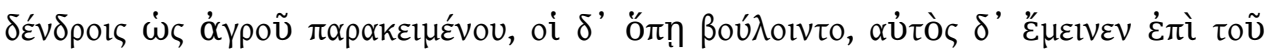

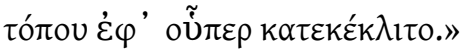

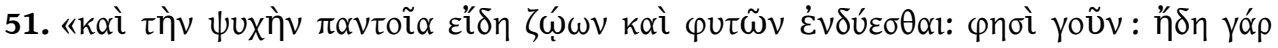

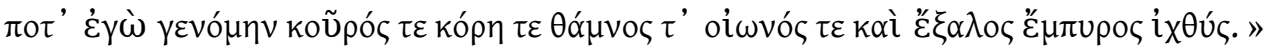

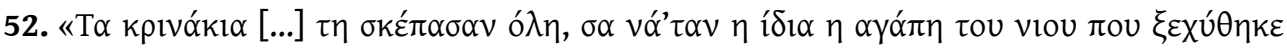

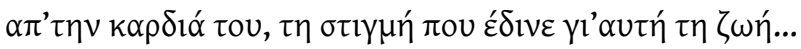

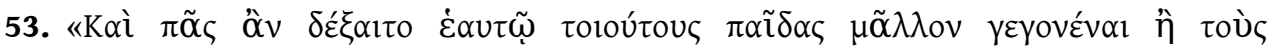

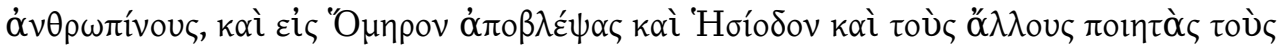






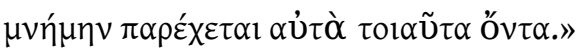

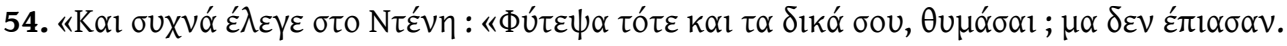

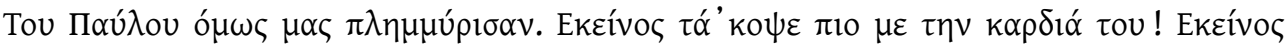

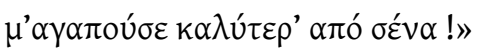

\section{RÉSUMÉS}

Le roman Anadyoméni de Grigorios Xénopoulos consiste en un dense réseau d'allusions à des repas, d'ordre littéraire, philosophique ou liturgique, qui dressent le décor d'une mise en pratique de la discussion, dans le Banquet de Platon, à propos de l'immortalité, du don de sa vie pour son amant, mais aussi de l'idée empédocléenne de la métempsycose sous la forme d'une plante.

Xenopoulos' novel Anadyomeni presents a dense network of literary, philosophical and liturgical references to meals, which set the stage for the enactment of the discussion, in Plato's Symposium, about immortality and giving one's life for one's lover, and of Empedocles' idea of metempsychosis in the form of a plant.

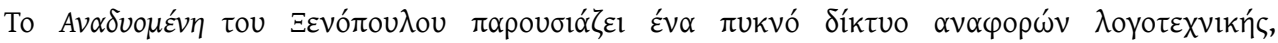

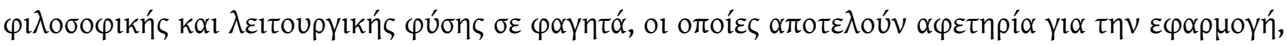

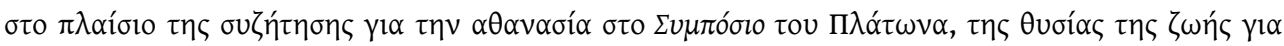

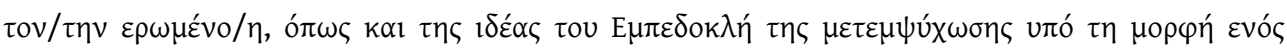

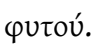




\section{INDEX}

Index géographique : Grèce, îles Ioniennes, Zante

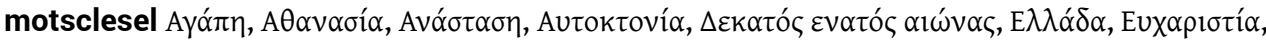

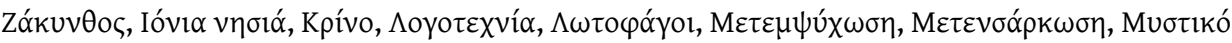

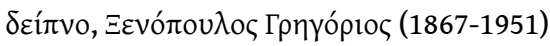

motsclesmk ЈОНСКИТЕ ОСТРОВИ, ЉУБОВ, БЕСМРТНОСТ, ВОСКРЕСЕНИЕ, ГРЦИЈА, ДЕВЕТНАЕСЕТТИОТ ВЕК, ЕВХАРИСТИЈАТА, ЗАКИНТОС, КСЕНОПУЛОС ГРИГОРИС (1867-1951), ЛИТЕРАТУРА, ЛОТУС ЈАДАЧИТЕ, МЕТЕМПСИХОЗА, РЕИНКАРНАЦИЈА, СОЛОМОС ДИОНИС (1798-1857), ТАJНАТА ВЕЧЕРА

Mots-clés : amour, Cène, Cène, Eucharistie, Eucharistie, immortalité, immortalité, Lotophages, Lotophages, métempsycose, métempsycose, réincarnation, réincarnation, résurrection, résurrection, Solomos Dionysios (1798-1857), Solomos Dionysios (1798-1857), Xénopoulos Grigoris (1867-1951), Xénopoulos Grigoris (1867-1951)

motsclestr Aşai rabbani ayini, Aşk, Diriliş, Edebiyat, İyonya adaları, Lotus Yiyenler, Ölümsüzlük, Ondokuzuncu yüzyıl, Reenkarnasyon, Ruh geçişmesi, Son akşam yemeği, Yunanistan, Zakyntos Keywords : Eucharist, Immortality, Lotus eaters, Love, Last Supper, Resurrection, Solomos Dionysios (1798-1857), Metempsychosis, Nineteenth century, Reincarnation, Ionian Islands, Greece, Xenopoulos Grigorios (1867), Literature

Thèmes : Littérature

\section{AUTEUR}

GUNNAR DE BOEL

Université de Gand 